\title{
A Reemergent Case of COVID-19 - Harbin City, Heilongjiang Province, China, April 9, 2020
}

\author{
Jun $\mathrm{Xu}^{1, .8}$; Yong Zhang ${ }^{2,3,8}$; Xiang Zhao ${ }^{2,3,8}$; Dayan Wang ${ }^{2,3}$; Weiping Dai'; Guangyu Jiao'; Shiwen Wang, \\ Jun $\mathrm{Liu}^{2,3}$; Weifeng Shi'; Cao Chen ${ }^{2,3}$; Shuhong Chen'; Shichun Yan'; Hua Hua'; George F. Gao ${ }^{1,3,5}$; \\ Wenbo $\mathrm{Xu}^{2,3, * ;}$; Guizhen $\mathrm{Wu}^{2,3, *}$
}

As of February 22, a total of 198 confirmed COVID-19 cases were reported in Harbin, Heilongjiang Province, and no new local cases were reported in Harbin over the next 54 days (1). However, on April 9, a newly confirmed COVID-19 case was reported with an unknown source of infection (2). The patient, a 54-year-old male, developed a fever on March 28 with the highest recorded temperature at $37.5{ }^{\circ} \mathrm{C}$. On April 7, the symptoms worsened and included dyspnea, cough, sputum, chest pain, and other symptoms, though he did not experience chest distress, heart palpitations, nausea and vomiting, abdominal pain or diarrhea. He went to the fever clinic of the Harbin Second Hospital and was admitted to the isolation ward with a temperature of $37.2^{\circ} \mathrm{C}$. The patient also had a history of diabetes.

The results of a routine blood examination were normal (WBC $5.15 \times 10^{9} / \mathrm{L}$; lymphocyte $1.66 \times 10^{9} / \mathrm{L}$, lymphocyte percentage $32.3 \%$; neutrophil percentage $51.9 \%$, c-reactive protein $36.56 \mathrm{mg} / \mathrm{L})$. Chest computed tomography (CT) showed multiple lymph node shadows in the mediastinum and multiple lamellar ground glass density shadows and mesh shadows in both lungs. On April 7, the throat swabs of the patients were tested by Harbin CDC by using realtime reverse transcription-polymerase chain reaction (real-time RT-PCR) targeting the ORF $1 \mathrm{ab}$ and $\mathrm{N}$ genes of COVID-19 virus, and the results showed that the nucleic acid of COVID-19 virus of the throat swab was positive. On April 8, Harbin CDC tested his pharyngeal swabs, blood, urine and stool samples again. COVID-19 nucleic acids were detected, but the test for serum antibodies for COVID-19 was negative.

The National Institute for Viral Disease Control and Prevention (IVDC) of China CDC conducted wholegenome sequencing analysis on a pharyngeal swab sample of the patient, which showed $99.97 \%$ nucleic acid similarity to the reference strain EPI_ISL_402119, which was isolated from Wuhan on January 7, 2020.
There was a total of eight nucleotide mutations between them. This strain possessed the nucleotide mutations C241T, C1059T, C3037T, C14408T, A23403G, and G25563T, which were consistent with the characteristics of the North American branch of the L lineage from Europe (Figure 1) (3). In addition, sequence alignment also revealed two mutations $\mathrm{C} 26636 \mathrm{~T}$ and C27213T in the virus genome from this patient. Among them, C1059T, C14408T, A23403G, and G25563T caused amino acid substitutions, which were located on 5'-UTR, ORF1ab, S protein, and ORF3a, respectively. This means that despite amino acid mutations in the virus, its biological characteristics, including its ability to bind to its receptor have not changed significantly.

Epidemiological investigations showed that the patient had no travel, residence, or stay history in Wuhan City and surrounding areas or other communities with confirmed COVID-19 case 14 days before the onset of disease (March 14-March 28). There was no contact with people with fever or respiratory symptoms from Wuhan City and surrounding areas nor from communities with confirmed case reports, nor did the patient have a history of overseas travel.

However, because this patient did not seek medical treatment and isolation immediately after the onset of fever, he still had normal social activities including holding a dinner party. Due to the prevalence of asymptomatic COVID-19 infection, epidemiological investigation and virus tracking surrounding this case became more challenging. Although the local transmission of the COVID-19 virus in the mainland of China had been interrupted, COVID-19 is still an epidemic in many other countries. Many cases of COVID-19 and asymptomatic infections entering China through airports and land ports have been detected (4). Therefore, it is necessary to strengthen COVID-19 surveillance in respiratory tract and stool 


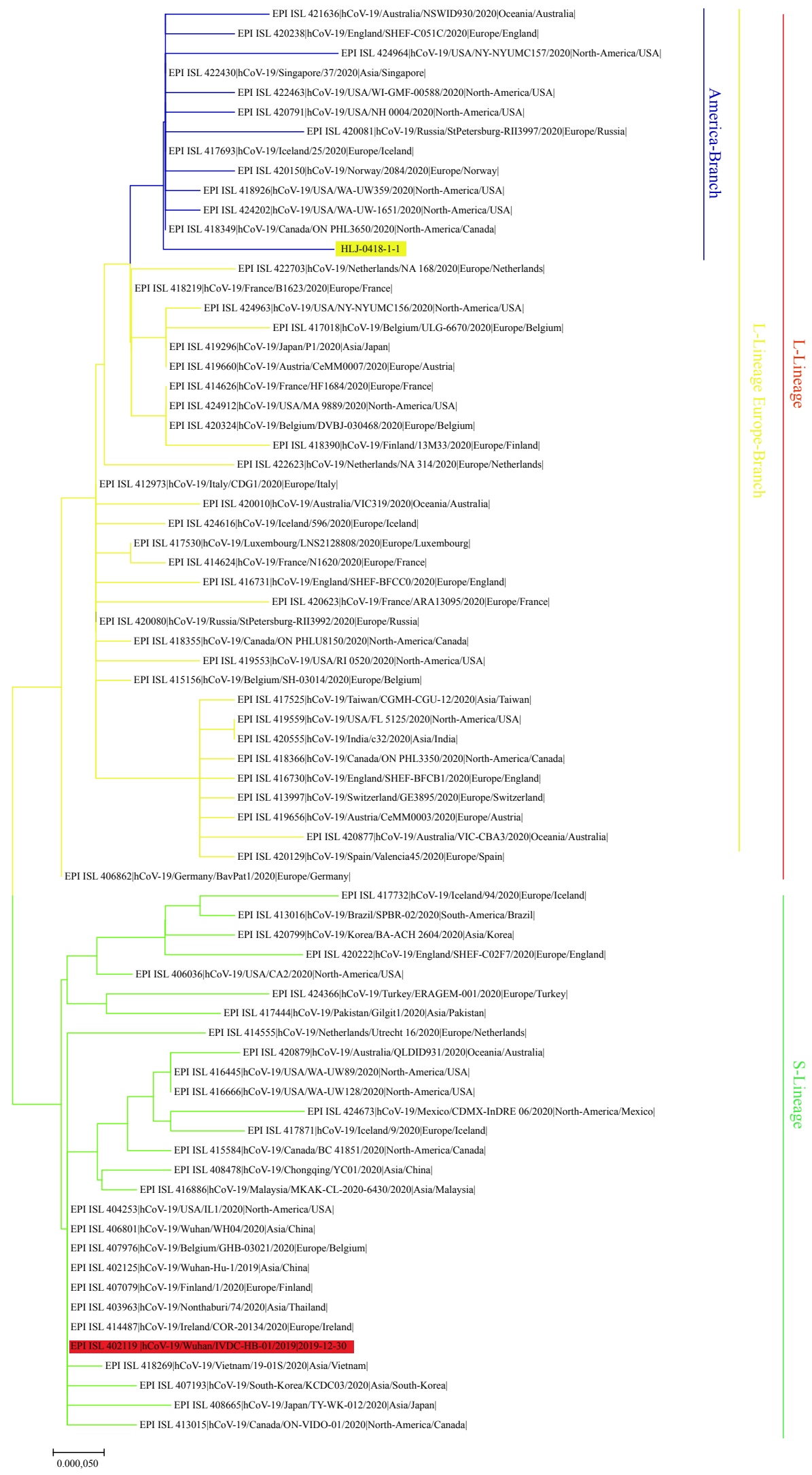

FIGURE 1. Phylogenetic tree based on the genome sequences of COVID-19 virus. The genome of COVID-19 virus of the case in Harbin City and Wuhan City were highlighted in shades of yellow and red, respectively. S- or L-lineage of COVID-19 virus were marked and colored on the right. 
specimens among overseas visitors.

Fundings: This work was supported by National Key Research and Development Program of China (Program No. 2018YFC1200305), National Science and Technology Major Project of China (Project No. 2018ZX10102001, 2018ZX10711001, 2018ZX1071 3002, 2017ZX10104001)

doi: $10.46234 / \mathrm{ccdcw} 2020.127$

* Corresponding authors: Wenbo Xu, xuwb@ivdc.chinacdc.cn; Guizhen Wu,wugz@ivdc.chinacdc.cn.

\footnotetext{
${ }^{1}$ Heilongjiang Center for Disease Control and Prevention, Harbin, Heilongjiang, China; ${ }^{2}$ National Health Commission Key Laboratory for Medical Virology, National Health Commission Key Laboratory for Biosafety, National Institute for Viral Disease Control and Prevention, Chinese Center for Disease Control and Prevention, Beijing, China; ${ }^{3}$ Center for Biosafety Mega-Science, Chinese Academy of Sciences, Wuhan, Hubei, China; ${ }^{4}$ Key Laboratory of Etiology and Epidemiology of Emerging Infectious Diseases in Universities of Shandong, Shandong First Medical University \& Shandong Academy
}

of Medical Sciences, Taian, Shandong, China; ${ }^{5}$ Chinese Center for Disease Control and Prevention, Beijing, China.

\& Joint first authors.

Submitted: June 12, 2020; Accepted: June 15, 2020

\section{REFERENCES}

1. Harbin Municipal People's Government. COVID-19 Situation in Harbin, 22 February 2020. http://www.harbin.gov.cn/art/2020/2/ 23/art_98_877381.html. [2020-02-22]. (In Chinese).

2. Harbin Municipal People's Government. COVID-19 Situation in Harbin, 9 April 2020. http://www.harbin.gov.cn/art/2020/4/10/art 98_892639.html. [2020-04-9]. (In Chinese).

3. Tang XL, Wu CC, Li X, Song YH, Yao XM, Wu XK, et al. On the origin and continuing evolution of SARS-CoV-2. Natl Sci Rev 2020. http://dx.doi.org/10.1093/nsr/nwaa036.

4. Li ZJ, Chen QL, Feng LZ, Rodewald L, Xia YY, Yu HL, et al. Active case finding with case management: the key to tackling the COVID-19 pandemic. Lancet 2020. http://dx.doi.org/10.1016/S0140-6736(20) 31278-2. 